\title{
Inventaire de la flore bactérienne dominante des Camemberts fabriqués avec du lait cru
}

\author{
par \\ J. RICHARD et Halima ZADI*
}

\section{Résu mé}

La flore bactérienne de Camemberts fabriqués avec du lait cru dans cinq usines différentes a été analysée à deux reprises, l'une en hiver, l'autre en été. Les prélèvements ont été réalisés à la surface et dans la masse des fromages, à différents stades de leur affinage.

La fraction dominante de la flore bactérienne non lactique, localisée essentiellement à la surface des fromages, était composée de bactéries corynéformes (dont une partie identifiable à Brevibacterium linens) de microcoques (Micrococcus et surtout Staph. saprophyticus) et d'entérobactéries, avec une très nette domination de l'espèce Hafnia alvei; Escherichia coli ne représentait que $9 \%$ de la flore coliforme. Des bactéries présentant des caractères intermédiaires entre les genres Moraxella et Acinetobacter ont été trouvées à un niveau élevé (plus de $10^{6} / \mathrm{g}$ ) dans tous les fromages et constituaient, en fin d'affinage, la flore dominante dans ceux d'une usine.

De grandes différences dans la nature de cette flore bactérienne non lactique ont été mises en évidence entre usines, mais pas aussi nettement, d'une saison à l'autre, pour une même usine.

Mots clés :

Camemberts - Lait cru - Flore bactérienne.

Titre abrégé :

Flore bactérienne des Camemberts de lait cru.

Laboratoire de Microbiologie Laitière et de Génie Alimentaire, I.N.R.A.-C.N.R.Z. 78350 Jouy-en-Josas (France).

Université de Constantine (Algérie) 


\section{S u m mary}

\section{DOMINANT BACTERIAL FLORA OF CAMEMBERT CHEESE MADE FROM RAW MILK}

The bacterial flora of Camembert cheese made from raw milk in five manufactures was determined twice, one in winter and the other in summer. The surface and the core of cheese at different stage of ripening were analysed separately.

The dominant part of the non lactic bacterial flora was mainly located on the surface of cheese and was composed of coryneform bacteria, comprising Brevibacterium linens, Micrococaecae (belonging maily to the genus Staph. saprophyticus) and Enterobacteriacae, with Hafnia alvei as dominant species. Escherichia coli and coliforms bacteria of minor importance were present at a lower level $(<10 \%$ of isolates of this group). Bacteria intermediate between the genera Moraxella and Acinetobacter were also found on the surface of all cheese at a significant level $\left(>10^{6} / \mathrm{g}\right)$; they were predominant on cheese from one manufacture by the end of the ripening period.

Large variations in flora composition were observed between but not to the same extend within manufactures.

Key words :

Camembert cheese - Raw milk - Bacterial flora.

\section{INTRODUCTION}

De nos jours, les fabrications de type traditionnel de Camemberts se distinguent des fabrications industrielles par divers aspects, dont l'utilisation de lait cru, considérée comme déterminante sur le plan des caractères des fromages obtenus (aspect, texture et surtout qualités gustatives).

L'examen bactériologique sommaire de ces fromages montre à l'évidence qu'ils possèdent, particulièrement à leur surface, une flore bactérienne se différenciant qualitativement et quantitativement de celle que l'on trouve dans les fromages de fabrication industrielle (tab. 1). Cette différence pourrait être la cause principale de celle que l'on reconnaît généralement, sur le plan organoleptique, entre les deux types de produits. Des variations qualitatives et/ou quantitatives de la flore bactérienne des Camemberts de lait cru peuvent également expliquer les différences de goût entre fromages de diverses origines ou, pour une même origine, d'une saison à l'autre. Il est donc particulièrement intéressant de connaître la nature et surtout les caractéristiques technologiques de cette flore : écologie (origine et condition de multiplication) et action sur les composés du fromage (modification de sa texture et apparition d'arôme). 
TABLEAU $1-T A B L E 1$

Analyse bactériologique de la surface de 25 Camemberts affinés (12 fabriqués avec du lait cru et 13 avec du lait pasteurisé) prélevés à la même époque de l'année*

Bacteriological analysis of 25 ripened Camembert cheese (12 made from raw milk and 13 from pasteurized milk) taken both on the market at the same period of the year*

\begin{tabular}{|c|c|c|c|}
\hline \multirow{2}{*}{ Groupe microbien } & \multirow{2}{*}{$\begin{array}{l}\text { Niveau de population } \\
\text { (par g de croûte) }\end{array}$} & \multicolumn{2}{|c|}{ Nombre d'échantillons } \\
\hline & & Camemberts de lait cru & Camemberts pasteurisés \\
\hline Bactéries lactiques & $>10^{8}$ & $9 / 12$ & $1 / 13$ \\
\hline Bactéries coliformes & $>10^{7}$ & $10 / 12$ & $1 / 13$ \\
\hline Bactéries pigmentées (a) & $>10^{6}$ & $9 / 12$ & $4 / 13$ \\
\hline Autres bactéries non pigmentées (b) & $>10^{7}$ & $9 / 12$ & $0 / 13$ \\
\hline
\end{tabular}

(a) Donnant principalement des colonies oranges (mostly orange colonies).

(b) Dont (including) Hafnia alvei.

* Ensemencement à la surface des milieux de culture utilisés (Spiral surface plating). 
La première étude microbiologique d'un fromage à pâte molle (le Brie) est due à Duclaux (1883). Il a montré la succession des principaux groupes de micro-organismes au cours de l'affinage de ce fromage : bactéries lactiques, levures, moisissures, «tyrothrix ». Mazé $(1905,1910)$ a confirmé ces résultats et a postulé que le rôle des "ferments " lactiques dans l'affinage était plus important que celui des «ferments du rouge », ces derniers n'assurant qu'un rôle protecteur contre l'oxydation. Sansonnetti (1930) a également observé la succession des flores du Camembert, mais attribue aux ferments du rouge la production de la saveur caractéristique de ce fromage. Elle a isolé de cette flore spécifique une bactérie dont la description qu'elle en fait permet de la classer dans le groupe corynéforme. Quelques propriétés biochimiques et les conditions de culture de cette bactérie ont été étudiées.

Plus près de nous, Lenoir (1963) a suivi l'évolution de la flore microbienne de Camemberts normands, du démoulage à la $4^{e}$ semaine d'affinage. Il a montré que les levures, les bactéries coliformes et les bactéries halotolérantes étaient en nombres plus importants à la surface du fromage que dans la masse (respectivement plus de $10^{7}$ et moins de $10^{6}$ micro-organismes $/ g$ ). Par la suite, Lenoir et Auberger (1966) ont étudié la nature des levures et de la flore halotolérante de ces fromages. Cette dernière était constituée pour la plus large part de microcoques, mais des bactéries corynéformes et des streptocoques fécaux étaient présents à un niveau non négligeable. Ces auteurs ont constaté également un changement significatif $(P<0,01)$ de la nature des levures et des microcoques au cours de l'affinage.

Jacquet et Coiffier (1972) ont plus particulièrement étudié la nature des bactéries coliformes de Camemberts fabriqués avec du lait cru et avec du lait pasteurisé, mais ne précisent pas le stade d'affinage de ces fromages. Or, d'après Mourgues et al. (1977) la nature de la flore coliforme change également au cours de l'affinage des fromages.

On ne dispose finalement que d'informations fragmentaires et insuffisamment précises sur la flore bactérienne des Camemberts fabriqués avec du lait cru. De plus, Lenoir a utilisé, dans son étude, des milieux de culture sélectifs et a pu, de ce fait, perdre une partie de la flore présente dans les fromages, comme le suggèrent les résultats des numérations obtenues par cet auteur : en effet, la somme des divers groupes microbiens dénombrés est significativement inférieure (d'au moins $10^{8}$ bactéries/g) au niveau atteint par la flore totale.

Cette étude a porté également sur les fabrications d'une seule usine à une époque où la traite manuelle était encore très pratiquée et le refroidissement du lait à la ferme quasi inexistant.

L'évolution relativement récente des conditions de production du lait (traite mécanique, refroidissement du lait à la ferme, paiement du lait suivant sa qualité) a pu entraîner une modification de sa flore naturelle et par conséquent, de celle des fromages ; d'autre part, l'existence possible de variations saisonnières et/ou d'une usine à 
l'autre nous ont incité à reprendre l'étude de la flore bactérienne des Camemberts de lait cru encore existants en y intégrant celle du lait de fabrication.

Une telle entreprise nécessite cependant la mise au point de milieux de culture appropriés, pour ne pas perdre des bactéries intéressantes, et de méthodes d'identification et/ou de caractérisation adaptables à l'examen d'un grand nombre de souches appartenant à des genres parfois mal définis (bactéries corynéformes par exemple). Le présent article est limité à un premier inventaire de la flore bactérienne dominante de Camemberts de lait cru à divers stages de leur affinage. Cette étude était destinée principalement à obtenir une collection de souches représentatives, indispensable à la mise au point de ces méthodes.

\section{MATERIEL ET METHODES}

\section{Origine et traitement des échantillons}

Cinq usines, réparties dans les principales zones de production de Camembert normand, ont été sélectionnées. Les fromages de deux fabrications, l'une en hiver (19-3-80), l'autre en été (11-6-80) ont été analysés aux stades suivants : au démoulage, après 6 jours, puis toutes les semaines jusqu'à la $6^{\mathrm{e}}$ comprise. A chaque stade, un fragment de la croûte (environ $3 \mathrm{~mm}$ d'épaisseur) et de coeur de trois fromages ont été prélevés séparément à l'aide d'une spatule stérile. Après le prélèvement de la croûte, les fromages ont été rompus en deux fractions de façon à éviter la contamination de la masse par les micro-organismes entraînés de la surface vers l'intérieur, comme cela peut se produire quand ils sont coupés avec un couteau. Au total, à chaque stade d'affinage, $5 \mathrm{~g}$ de coeur et $5 \mathrm{~g}$ de croûte provenant de trois fromages par usine ont été prélevés et malaxés avant d'être introduits dans un flacon contenant $45 \mathrm{ml}$ d'une solution stérile de citrate de sodium à $2 \%$. Cette suspension a été ensuite homogénéisée à l'aide d'une turbine (appareil Ultra-Turrax, type TP 18/2) tournant à $20000 \mathrm{t} / \mathrm{min}$. Ce traitement est également destiné à assurer une dispersion maximale des amas microbiens (Richard, 1980 ; Richard et al., 1981). Une dilution finale à $1 / 100$ dans une solution stérile de citrate à $2 \%$ de cette suspension homogénéisée sert à ensemencer les boîtes de Petri.

\section{Dénombrements microbiens}

a) Milieux et conditions de culture

Pour le dénombrement des bactéries à Gram positif, après l'essai comparatif de plusieurs milieux nutritifs de base, nous avons retenu la gélose nutritive déjà utilisée par Lenoir, car elle permettait à la 
fois une bonne croissance des colonies et le développement optimum de la pigmentation de certaines d'entre elles. Ce milieu a la composition suivante (par litre) : extrait de levure $3 \mathrm{~g}$, extrait de viande $5 \mathrm{~g}$, tryptone $10 \mathrm{~g}$, glucose $5 \mathrm{~g}$, gélose $15 \mathrm{~g}$. Nous y avons ajouté de l'acide nalidixique $(40 \mathrm{mg} / \mathrm{l})$ pour éliminer les bactéries à Gram négatif (Beerens et Tahon-Castel, 1966), de l'amphotéricine B (10 mg/l) pour empêcher la croissance des levures et des moisissures (Zadi, 1981) et du phosphate disodique $(5 \mathrm{~g} / 1)$. Trois $\mathrm{pH}$ ont été comparés : $\mathrm{pH} 7,0$, $\mathrm{pH} 8,0$ et $\mathrm{pH} 9,0$.

Nous avons également utilisé le milieu hypersalé de Chapman (Mannitol Salt Agar, Difco) pour comparer nos résultats à ceux de Lenoir, en ce qui concerne la flore halotolérante.

Les bactéries coliformes ont été dénombrées sur milieu lactosé au cristal violet et bile (Violet Red Bile Agar (VRB), Difco) (Anon. 1974).

\section{b) Ensemencement des milieux et comptage des colonies}

Les suspensions de fromages (surface et cœur) ont été ensemencées à la surface de la gélose répartie en boîtes de Petri de $140 \mathrm{~mm}$ de diamètre, à l'aide d'un ensemenceur Spiral Plater*. Cette méthode permet de faire des dénombrements microbiens dans les rapports de 1 à 1000 (soit, dans notre cas, de $10^{6}$ à $10^{9}$ micro-organismes par gramme de fromage) avec une seule boîte de Petri. L'ensemencement en surface assure en outre une différenciation immédiate des colonies suivant leur taille, leur aspect et leur pigmentation. Les colonies des micro-organismes de la flore dominante sont également bien séparées, permettant ainsi un prélèvement ultérieur sans risque de contamination par des colonies voisines comme cela se produit souvent avec la méthode classique d'ensemencement dans la masse de la gélose.

Pour la flore à Gram positif, les boîtes de Petri ont été incubées en aérobiose à $20-30^{\circ} \mathrm{C}$, pendant $48 \mathrm{~h}$, puis à température ambiante pendant environ 15 jours, pour permettre le développement maximum des colonies et de leur pigmentation. Leur comptage a été fait manuellement. Pour cela on a dessiné sur le fond des boîtes de Petri quatre couronnes concentriques de $13,5 \mathrm{~mm}$ de largeur auxquelles correspondent des volumes connus d'inoculum ; on a choisi pour le comptage la couronne comportant des colonies bien isolées et en nombres inférieures à 100 . On y a effectué une numération des divers types de colonies se différenciant par l'aspect (morphologie, pigmentation, opacité).

A l'usage, les principaux types observés ont été les suivants : les petites colonies (diamètre inférieur à $2 \mathrm{~mm}$ ), plates, translucides, non pigmentées, correspondaient essentiellement aux streptocoques lactiques (Richard 1982); les autres bactéries formaient des colonies de

* Interscience, 3, rue de Milan - 75009 Paris. 
diamètre nettement supérieur à celui des bactéries lactiques ; suivant leur pigmentation, on a pu différencier les types A (oranges opaques), B (non pigmentées opaques), C (jaunes opaques) et D (autres aspects, généralement colonies non pigmentées translucides).

Pour une raison essentiellement pratique (ensemencement en spirale à la surface de la gélose), le milieu de dénombrement des bactéries coliformes n'était pas recouvert d'une seconde couche comme habituellement recommandé. Après $24 \mathrm{~h}$ d'incubation à $30^{\circ} \mathrm{C}$, on a dénombré séparément les colonies acidifiantes et celles qui ne l'étaient pas.

\section{Classification et identification de la flore dominante}

a) Flore à Gram positif non lactique

On a prélevé, sur la gélose nutritive tamponnée (GNT) à 7,0 ou à pH 8,0 et sur milieu MSA (Mannitol Salt Agar), une colonie de chaque type $\mathrm{A}, \mathrm{B}, \mathrm{C}$ ou $\mathrm{D}$ lorsqu'il correspondait à une population supérieure à $10^{6} / \mathrm{g}$ de fromage. On a ainsi constitué une collection de 162 souches qui, après purification, ont été classées sur la base des critères suivants :

- mobilité et morphologie à l'état frais sur culture jeune (24 à 48 h) et sur culture âgée (1 semaine) de façon à mettre en évidence un éventuel changement (Mulder et Antheunisse 1963) ; les cultures étaient réalisées sur GNT à pH 7,0 non gélosé ;

- coloration de Gram sur culture jeune ;

- culture en présence d'acide nalidixique (40 mg/1) et de pénicilline $(100 \mathrm{UI} / \mathrm{ml})$, sur milieu BHI (Brain Hart Infusion Agar, Difco) et sur MSA.

Les micro-organismes des différents groupes formés sur la base de la morphologie, de la coloration de Gram et de la résistance aux deux antibiotiques ont été ensuite identifiés autant que possible au rang du genre ou de l'espèce par des méthodes classiques ou par analyse numérique, comme il sera indiqué plus loin.

b) Bactéries coliformes et bactéries lactose négatif sur milieu VRB

$\mathrm{Au}$ total 79 colonies acidifiantes et 25 bactéries lactose négatives, ont été prélevées au hasard sur le milieu VRB parmi la flore dominante des fromages à divers stades de leur affinage. Après purification, les souches ont été soumises aux tests suivants : morphologie des cellules à l'état frais, coloration de Gram, mode d'utilisation du glucose par la méthode à un tube (Park et Holding 1966). Toutes les souches pouvant être classées comme entérobactéries, elles ont été soumises à une identification à l'aide des galeries API 20E* .

\footnotetext{
* API System S.A., La Balme-les-Grottes - 38390 Montalieu-Vercieu (France).
} 


\section{RESULTATS ET DISCUSSION}

\section{Comparaison des milieux}

On constate (tab. 2) que le milieu de Chapman donne, pour la flore non lactique, pratiquement les mêmes résultats que la gélose nutritive tamponnée (GNT) à $\mathrm{pH} \mathrm{7,0}$ ou $\mathrm{pH} 8,0$ mais ne permet pas, en général, le développement des bactéries lactiques. Parfois cependant, du fait de son acidification par le développement de certaines colonies (probablement des staphylocoques), ce milieu ne permettait pas la croissance des colonies oranges. On a constaté en effet (Zadi, 1981) que ces dernières étaient, en présence de 5 à $10 \%$ de sel, très sensibles à un abaissement de $\mathrm{pH}$ au-dessous de la neutralité, ce qui confirme les observations de Mulder et al. (1966).

\section{Evolution des différents groupes microbiens au courș de l'affinage}

\section{a) Flore lactique}

Les résultats des dénombrements des bactéries lactiques sont présentés dans le tableau 3. N'ayant pas observé de variations de niveau de cette flore au cours de l'affinage, nous avons effectué une moyenne des résultats des six dénombrements par usine (une par semaine).

Comme l'a déjà observé Lenoir (1963), la flore lactique représentait la majeure partie de la flore à Gram positif aussi bien à l'intérieur que sur la croûte du fromage (environ $10^{9} / \mathrm{g}$ ). Le nombre de bactéries lactiques dans la croûte n'était pas significativement différent de celui trouvé dans la masse du fromage $(\mathrm{P}<0,05)$. Par contre, on a observé dans toutes les usines, une différence significative dans les nombres moyens de bactéries lactiques entre la première et la deuxième fabrication : dans cette dernière, la population lactique était près de 10 fois plus importante que dans la première.

b) Flore non lactique

L'évolution de la flore bactérienne non lactique poussant sur le milieu de Chapman ou le milieu GNT des Camemberts fabriqués en été est présentée sur la figure 1. La flore interne étant en général à un niveau nous paraissant trop faible (moins de $10^{6}$ cellules par gramme) pour avoir un rôle dans l'affinage de ces fromages, nous n'y avons pas fait figurer son évolution. Les valeurs qui ont servi à établir les courbes correspondent à la moyenne des numérations sur trois milieux (MSA, GNT à pH 7,0 et $\mathrm{pH} 8,0$ ) de façon à avoir plus de précision. On a regroupé les nombres de colonies non pigmentées (types B et D) car suivant les milieux, il était parfois difficile de séparer celles qui étaient opaques de celles qui ne l'étaient pas. On 


\section{TABLEAU 2 - TABLE 2}

Comparaison du milieu de Chapman (MSA) et de la gélose nutritive tamponnée additionnée d'acide nalidixique (40 mg/l) et d'amphotéricine $\mathrm{B}(10 \mathrm{mg} / \mathrm{l})$, à $3 \mathrm{pH}$ différents

Comparison of 4 media : MSA (Mannitol Salt Agar),

GNT (yeast extract - peptone - glucose agar containing nalidixic acid and amphotericin B) at pH 7, 8 and 9

\begin{tabular}{|c|c|c|c|c|c|}
\hline Groupe bactérien & $\mathrm{N}$ & \multicolumn{4}{|c|}{ Classement des milieux par ordre de numérations croissantes } \\
\hline \multicolumn{6}{|l|}{ Flore lactique : } \\
\hline - masse (core) & 19 & MSA $(<4,5)$ & pH $9(8,25)$ & pH $8(8,50)$ & pH $7(8,69)$ \\
\hline - croûte (surface) & 19 & MSA $(<4,5)$ & pH $9(8,51)$ & pH $7(8,76)$ & pH $8(8,88)$ \\
\hline \multicolumn{6}{|l|}{ Flore non lactique* : } \\
\hline - colonies oranges opaques & 18 & pH $9(6,57)$ & pH $7(7,29)$ & $\mathrm{pH} 8(7,53)$ & MSA $(7,84)$ \\
\hline - colonies non pigmentées & 7 & MSA $(8,46)$ & pH $9(8,53)$ & pH $7(8,54)$ & pH $8(8,62)$ \\
\hline - colonies jaunes opaques & 8 & pH $9(5,60)$ & pH $7(5,99)$ & MSA $(6,01)$ & pH $8(6,43)$ \\
\hline
\end{tabular}

N : Nombre d'échantillons de fromages.

Entre parenthèses : Logarithmes du nombre moyen de bactéries par gramme de fromage.

* : Flore de surface essentiellement.

Les moyennes réunies par un trait ne diffèrent pas significativement au seuil $\mathbf{P}=0,05$. 
TABLEAU 3 - TABLE 3

Niveau de la flore lactique dans les fromages (moyenne log arithmique du nombre de bactéries lactiques / g de fromage, de la semaine 1 à la semaine $6 \pm$ intervalle de confiance à $\mathrm{P}=0,95$ )

Level of the lactic flora in the cheese (geometric mean of results from week 1 to 6)

\begin{tabular}{|c|c|c|c|c|}
\hline Usine & Saison & Croûte (surface) & Cour (core) & Moyenne (mean) \\
\hline I & $\begin{array}{l}\text { hiver } \\
\text { été }\end{array}$ & $\begin{array}{l}7,66 \pm 0,41 \\
\underline{8,37 \pm 0,13}\end{array}$ & $\begin{array}{l}7,67 \pm 0,50 \\
\underline{8,95 \pm 0,33}\end{array}$ & $\left.\begin{array}{l}7,66 \pm 0,32 \\
8,36 \pm 0,18\end{array}\right\} 12,69 * *$ (b) \\
\hline II & $\begin{array}{l}\text { hiver } \\
\text { été }\end{array}$ & $\begin{array}{l}\frac{8,05 \pm 0,28}{9,15 \pm 0,09} \\
\end{array}$ & $\begin{array}{l}\frac{8,19 \pm 0,21}{9,19 \pm 0,12} \\
\end{array}$ & $\left.\begin{array}{l}8,12 \pm 0,18 \\
9,17 \pm 0,078\end{array}\right\} 100,65^{* *}$ \\
\hline III & $\begin{array}{l}\text { hiver } \\
\text { été }\end{array}$ & $\begin{array}{l}\underline{8,34 \pm 0,21} \\
\underline{8,35 \pm 0,34}\end{array}$ & $\begin{array}{l}\frac{7,96 \pm 0,10}{\underline{8,63} \pm 0,4} \\
\end{array}$ & $\left.\begin{array}{l}8,15 \pm 0,16 \\
8,47 \pm 0,28\end{array}\right\}$ \\
\hline IV & $\begin{array}{l}\text { hiver } \\
\text { été }\end{array}$ & $\begin{array}{l}\frac{8,19 \pm 0,13}{9,09 \pm 0,2} \\
\end{array}$ & $\begin{array}{l}\underline{8,03 \pm 0,27} \\
\underline{8,89 \pm 0,37}\end{array}$ & $\left.\begin{array}{l}8,11 \pm 0,15 \\
8,99 \pm 0,22\end{array}\right\} 15,95^{* *}$ \\
\hline $\mathrm{V}$ & $\begin{array}{l}\text { hiver } \\
\text { été }\end{array}$ & $\begin{array}{l}\underline{7,65 \pm 0,17} \\
\underline{8,83 \pm 0,39} \\
\end{array}$ & $\begin{array}{l}\underline{7,74 \pm 0,37} \\
\underline{9,06 \pm 0,16}\end{array}$ & $\left.\begin{array}{l}7,69 \pm 0,2 \\
8,94 \pm 0,22\end{array}\right\} 19,38^{*}$ \\
\hline
\end{tabular}

(a) Les moyennes réunies par un trait ne diffèrent pas significativement au seul $P=0,05$ (test $t$ ).

(b) Comparaison de la première et de la deuxième fabrications de chaque usine : rapports de variances significatifs aux seuils $\mathrm{P}=0,05^{*}$ et $\mathrm{P}=0,01^{* * *}$. 

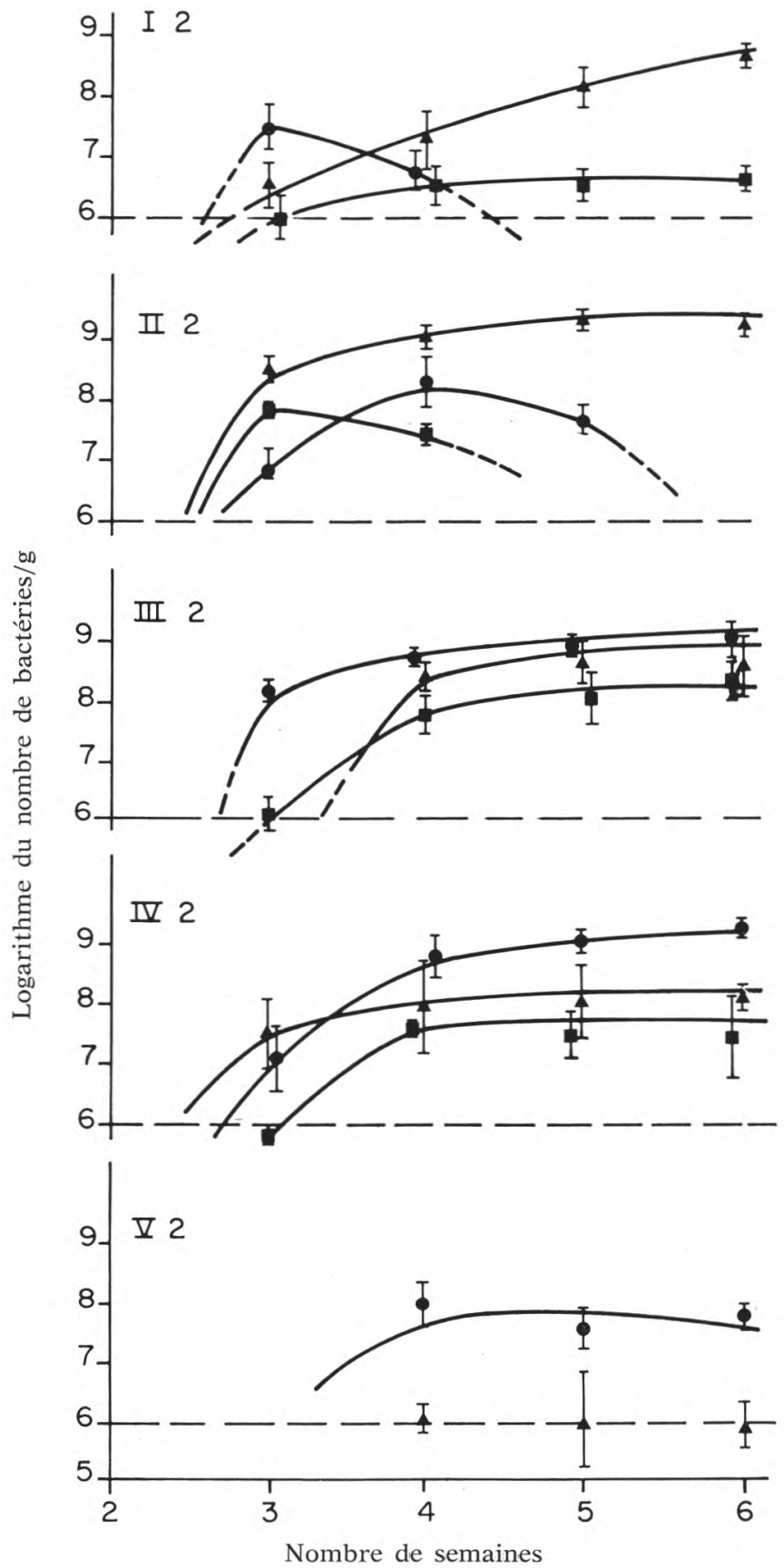

Fig. 1

Evolution de la flore non lactique poussant sur les milieux GNT et MSA trouvée dans les Camemberts de la fabrication d'été (moyenne de trois déterminations avec intervalle de confiance à $\mathrm{P}=95 \%$ ).

$$
\begin{aligned}
& \text { - : colonies oranges opaques ; } \\
& \text { - : colonies jaunes opaques ; } \\
& \text { : colonies non pigmentées. }
\end{aligned}
$$


peut faire les observations suivantes : dans les fabrications I et II, les colonies oranges opaques dominaient nettement en fin d'affinage, alors que dans les fabrications III et IV, on avait un mélange de trois types à peu près au même niveau. Dans l'usine V, la flore était assez particulière : les colonies oranges opaques restaient à un niveau faible et constant du début jusqu'à la fin de la maturation ; la flore dominante était constituée de bactéries donnant des colonies un peu opaques, non pigmentées, d'aspect tout à fait différent des colonies de même type observées avec les autres fromages.

Des résultats semblables, mais moins précis, ont été obtenus avec les fabrications d'hiver.

Pour la flore coliforme (colonies acidifiant le milieu VRB), comme Lenoir (1963) et contrairement à Mourques et al. (1977), nous n'avons pas observé, au cours de l'affinage des fromages, une évolution régulière de niveau. Les résultats moyens du nombre de bactéries coliformes et de bactéries lactose-négatives pour les deux fabrications de chaque usine sont présentés sur le tableau 4. On constate que la

\section{TABLEAU 4-TABLE 4}

Niveau de la flore à Gram négatif des fromages (culture sur milieu VRB) Level of Gram negative flora of cheese (enumeration on Violet Red Bile Agar)

\begin{tabular}{|c|c|c|c|c|c|c|}
\hline \multirow{3}{*}{\multicolumn{2}{|c|}{ Usine }} & \multirow{3}{*}{ Saison } & \multicolumn{4}{|c|}{ Nombre de bactéries $\times 106 / \mathrm{g}$} \\
\hline & & & \multicolumn{2}{|c|}{ Coliformes } & \multicolumn{2}{|c|}{ Bactéries lactose - } \\
\hline & & & surface (a) & masse (b) & surface (a) & masse (b) \\
\hline & I & $\begin{array}{l}\text { hiver } \\
\text { été }\end{array}$ & $\stackrel{21}{5,5}$ & $\begin{array}{r}2,0-5,9 \\
<0,1-0,3\end{array}$ & $\begin{array}{l}16 \\
10\end{array}$ & $\begin{array}{l}<0,1-2,0 \\
<0,1\end{array}$ \\
\hline & II & $\begin{array}{l}\text { hiver } \\
\text { été }\end{array}$ & $\begin{array}{l}9,8 \\
2,7\end{array}$ & $\begin{array}{l}<0,1-0,3 \\
<0,1-1,0\end{array}$ & $\begin{array}{l}25 \\
29\end{array}$ & $\begin{array}{l}<0,1-1,2 \\
<0,1-0,1\end{array}$ \\
\hline & III & $\begin{array}{l}\text { hiver } \\
\text { été }\end{array}$ & $\begin{array}{l}2,1 \\
1,0\end{array}$ & $\begin{array}{r}0,3-0,9 \\
<0,1-0,5\end{array}$ & $\begin{array}{l}32 \\
17\end{array}$ & $\begin{array}{l}<0,1-0,8 \\
<0,1-0,2\end{array}$ \\
\hline & IV & $\begin{array}{l}\text { hiver } \\
\text { été }\end{array}$ & $\begin{array}{r}7,2 \\
<0,2\end{array}$ & $\begin{array}{l}<0,1-1,1 \\
<0,1-0,9\end{array}$ & $\begin{array}{r}12 \\
4\end{array}$ & $\begin{array}{l}<0,1-1,8 \\
<0,1-5,5\end{array}$ \\
\hline & $\mathrm{V}$ & $\begin{array}{l}\text { hiver } \\
\text { été }\end{array}$ & $\begin{array}{l}1,6 \\
0,4\end{array}$ & $\begin{array}{l}<0,1-0,3 \\
<0,1-1,3\end{array}$ & $\begin{array}{l}19 \\
52\end{array}$ & $\begin{array}{l}<0,1 \\
<0,1-0,7\end{array}$ \\
\hline $\begin{array}{l}\text { (a) } \\
\text { (b) }\end{array}$ & \multicolumn{6}{|c|}{ 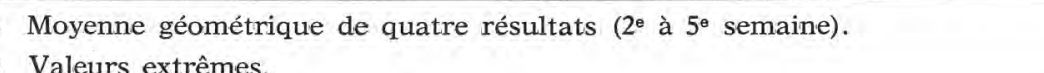 } \\
\hline
\end{tabular}


flore lactose + ou lactose - se développe beaucoup plus en surface du fromage que dans sa masse, ce qu'avait déjà observé Lenoir pour la flore coliforme. On observe par ailleurs que les bactéries lactosesont, en surface, à un niveau nettement supérieur à celui des bactéries lactose + .

\section{Caractérisation et identification des souches isolées}

Les résultats de l'étude cytologique et de la caractérisation primaire des 162 souches isolées du milieu MSA ou du milieu GNT à pH 7,0 ou $\mathrm{pH} 8,0$ sont présentés sur le tableau 5 . Les bactéries sous forme de cocci en culture jeune ont été rangées dans les microcoques ; la plupart étaient nettement Gram positif ; dans quelques cas toutefois, on avait un mélange de cellules positives et négatives. Presque toutes ces bactéries poussaient sur milieu MSA qu'elles acidifiaient sauf celles du groupe C. A l'exception de ces dernières, presque toutes poussaient en présence d'érythromycine. Les microcoques des types A, B et D peuvent donc être rangés dans le genre Staphylococcus, alors que ceux du type $\mathrm{C}$ appartiennent au genre Micrococcus (BairdParker 1979). Les premiers ne poussaient pas ou très mal en anaérobiose ; ils ne possédaient pas de coagulase ni de nucléase thermostable et étaient résistants à la novobiocine. Ils peuvent donc être identifiés à l'espèce $S$. saprophyticus ou à l'espèce voisine S. cohnii (BairdParker 1979).

Les bâtonnets de forme irrégulière, groupés en caractères chinois, en palissades ou formant des $\mathrm{V}$ caractéristiques ont été classés comme bactéries corynéformes (Rogosa et al., 1974) ; pour beaucoup d'entre elles, les cellules étaient sous forme cocci après 4 à 5 jours de culture. Le Gram était le plus souvent variable (mélange de cellules positives et négatives).

Les colonies de type B contenaient un tiers de levures isolées principalement du milieu GNT, où elles ont poussé malgré la présence d'un antibiotique spécifique; nous en avons trouvé autant après 4 semaines d'affinage qu'avant, et également sur les fromages provenant de toutes les usines. Comme il est normal pour des cellules eucaryotes, elles étaient résistantes à la pénicilline.

Les colonies de type $\mathrm{C}$ contenaient plus de bactéries corynéformes que de microcoques; comme celles du type $\mathrm{A}$, elles étaient isolées surtout à partir de la $4^{\mathrm{e}}$ semaine d'affinage et provenaient de la surface des fromages de trois usines sur quatre.

Dans les colonies du type D, nous avons trouvé surtout des bâtonnets courts ou des coccobacilles, immobiles, souvent Gram variable, tous sensibles à l'acide nalidixique, et des bâtonnets mobiles, Gram négatif, également sensibles à cet antibiotique, mais pour la plupart résistants à la pénicilline. Les premiers ont été identifiés comme étant du genre Acinetobacter ou Moraxella selon que les bactéries étaient oxydase-positives ou non et résistantes ou sensibles 
TABLEAU 5 - TABLE 5

Caractérisation primaire des 162 souches isolées des milieux GNT ( $\mathrm{pH} 7$ ou 8) et MSA

Primary characterization of strains isolated from GNT ( $p H 7$ or 8) and MSA

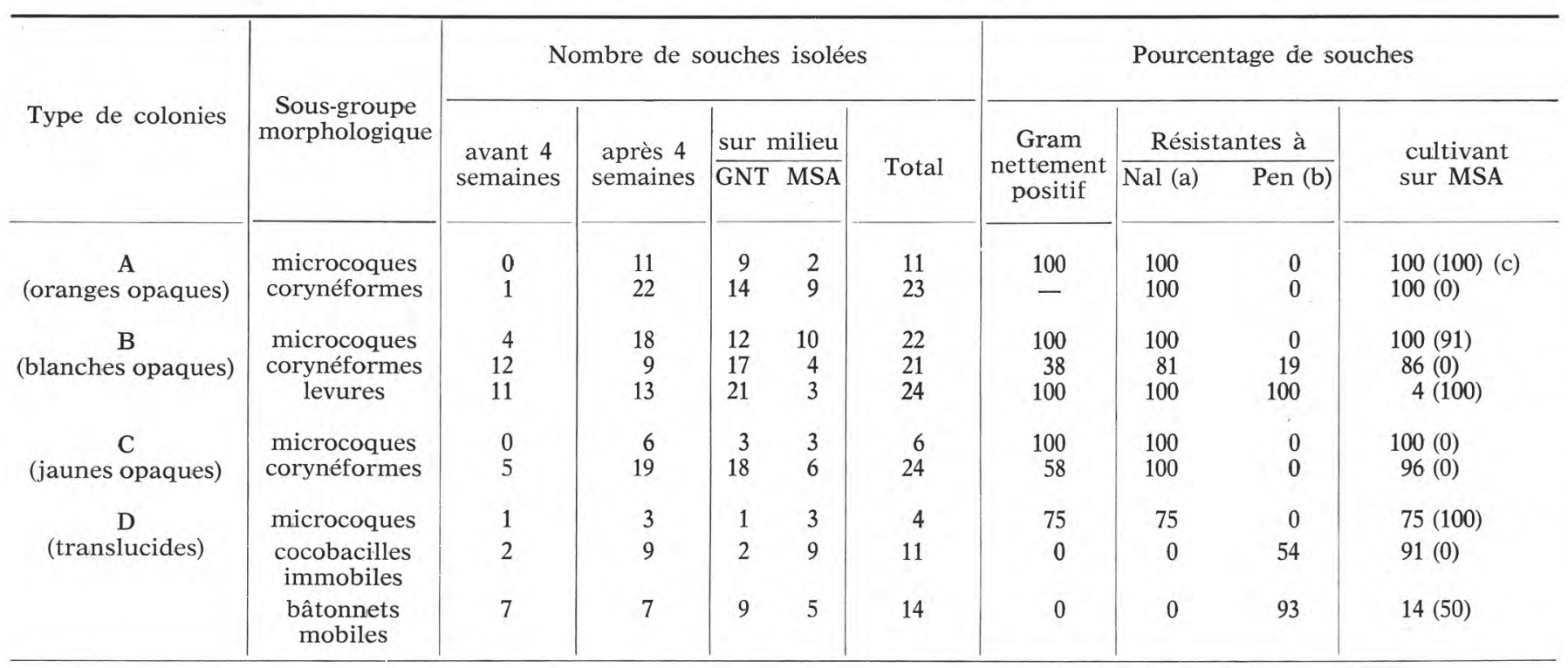

(a) Acide nalidixique $(40 \mathrm{mg} / \mathrm{l})$.

(b) Pénicilline G $(100 \mathrm{UI} / \mathrm{ml})$.

(c) Entre parenthèses : pourcentage de souches acidifiant le milieu (percentage of acidifying colonies). 
à la pénicilline (Reyn 1974). Ces Moraxella paraissent appartenir à l'espèce $M$. phenylpyruvica*. Les bâtonnets mobiles étaient des entérobactéries identifiables (voir plus loin) aux espèces E. coli ( 3 souches), Hafnia alvei (7 souches), Enterobacter agglomerans, Serratia liquefaciens (1 souche chacun) ; 2 souches n'ont pas pu être identifiées. D'une façon générale, les microcoques et les bactéries corynéformes étaient résistants à l'acide nalidixique et sensibles à la pénicilline. On a montré par ailleurs (Richard et Zadi, 1982) que les bactéries corynéformes du groupe A pouvaient être identifiées à Brevibacterium linens et qu'elles avaient des propriétés biochimiques nettement différentes des autres bactéries corynéformes ou des microcoques isolés des Camemberts.

Les 79 souches de coliformes isolées des fromages en cours d'affinage ont été identifiées à Hafnia alvei (51\%), E. coli $(9 \%)$, Ent. cloacae et Serratia liquefaciens ( $8 \%$ chacun). La plupart des autres souches n'ont pu être identifiées. Aucune variation nette dans les pourcentages n'a été observée entre la surface et le cœur du fromage ou au cours de l'affinage, ni entre les différentes fabrications. Toutefois le faible nombre de souches pour chaque stade ne permettait pas de voir avec précision de telles variations. Dans l'ensemble, ces résultats confirment ceux de Mourgues et al. (1977) ou de Tolle et al. (1980) qui ont observé dans les Camemberts pasteurisés une domination de Hafnia alvei (Ent. hafniae) sur l'ensemble des Enterobacteriaceae. Par contre, Jacquet et Coiffier (1973) ont trouvé E. coli aussi souvent que Hafnia alvei (40\% de la flore coliforme). alvei.

Les colonies lactose négatives ont été identifiées à l'espèce Hafnia

\section{CONCLUSIONS}

Comme on a pu le voir, la flore bactérienne non lactique présente principalement à la surface des Camemberts traditionnels est complexe. Nous avons pu mettre en évidence, à un niveau élevé, des groupes bactériens non encore observés sur ces fromages (Moraxella et Acinetobacter, par exemple) ou sous-estimés, comme les différents types de bactéries corynéformes. Dans l'étude de Lenoir, en effet, ces dernières étaient à un niveau inférieur à celui des microcoques. Cela est probablement dû au fait que cet auteur a examiné les fromages jusqu'à la $4^{\mathrm{e}}$ semaine d'affinage seulement alors que nous avons poursuivi notre étude 2 semaines de plus.

La présence, à un niveau élevé, de bactéries identifiables à l'espèce Brevibacterium linens est particulièrement intéressante. Ces bactéries sont probablement à l'origine d'une des composantes du goût spécifique des Camemberts traditionnels, en raison de leur aptitude à

* Nous remercions le Dr C. Richard, Institut Pasteur de Paris, qui a confirmé cette identification et l'a poursuivie au niveau de l'espèce. 
dégrader la méthionine avec production de méthane-thiol et de dérivés soufrés responsables du goût " aillé » de nombreux fromages (Dumont et al. 1976, Hemme et al. 1982). Cependant ceux de l'usine V, dont la flore était pauvre en ces bactéries, n'étaient pas moins riches en goût que ceux des autres usines. D'autres bactéries (ou micro-organismes) peuvent donc participer à la production de ce composé d'arôme ou à d'autres arômes.

D'après Henriksen (1973) et Lautrop (1974) les bactéries du genre Moraxella peuvent être parasites des muqueuses des animaux à sang chaud, ou seulement saprophytes. Les exigences nutritionnelles de ces bactéries sont également très grandes et leur optimum de culture est compris entre 33 et $37^{\circ} \mathrm{C}$. Ce ne sont pas des caractéristiques rencontrées avec les souches que nous avons isolées du Camembert. Elles se sont développées en effet au cours de l'affinage à une température comprise entre 3 et $6^{\circ} \mathrm{C}$ et par ailleurs, elles se cultivent aisément sur des milieux relativement simples. Elles ressemblent plus de ce fait, aux bactéries du genre Acinetobacter isolées du lait (Henriksen 1973, Lautrop 1974, Richard 1982). Dans ce genre, A. calcoaceticus a été signalé comme responsable d'un défaut de goût et d'aspect d'un fromage à pâte molle à la surface duquel cette espèce avait proliféré (Carini et al., 1978). A un niveau plus faible, ces bactéries pourraient, par contre, contribuer à la formation de composés d'arôme intéressants.

L'étude que nous avons entreprise n'est évidemment pas complète. Nous ne sommes pas sûrs d'avoir mis en évidence tous les groupes de bactéries présentes dans ce fromage à un niveau significatif (plus de $10^{6}$ cellules $/ \mathrm{g}$ ). Egalement, nous n'avons examiné, par notre mode de sélection des souches, que la flore dominante de chaque groupe observé ; par ailleurs, chacun d'eux subit peut-être lui-même une évolution qualitative au cours de l'affinage des fromages comme l'ont montré Lenoir et Auberger (1966) pour les levures et les microcoques. Nous avons pu observer des différences très nettes entre les fabrications étudiées mais pas encore, pour une même usine, d'une saison à l'autre.

Bien que cette étude mérite d'être complétée, compte tenu du volume de travail qu'elle nécessite, il paraît judicieux, dans un premier temps, de définir le rôle direct ou indirect, dans la production d'arôme, des différents groupes bactériens que nous avons déjà trouvés à un haut niveau dans les Camemberts. Puis, l'écologie des bactéries intéressantes (origine, relation avec le substrat ou avec les autres micro-organismes, effet de facteurs intrinsèques comme l'humidité, la salinité, le $\mathrm{pH}$, ou extérieurs, comme la température) devrait être étudiée soigneusement, car il n'apparaît pas que l'addition simple à du lait pasteurisé, de souches sélectionnées à partir de fromages traditionnels de bonne qualité gustative, comme le proposait déjà Maze en 1910, ait jusqu'à présent, amélioré considérablement la qualité des produits obtenus. 


\section{Remerciements}

Nous remercions vivement Jean-Jacques Gratadoux pour sa précieuse collaboration technique à l'étude, notamment en ce qui concerne la caractérisation des souches et leur entretien.

Nos remerciements vont également aux fabricants de Camemberts qui ont bien voulu participer à l'étude et nous ont procuré toutes facilités pour sa réalisation.

\section{Bibliographie}

ANONYME (1974). - Lait et produits laitiers. Dénombrement des coliformes. Méthode de routine $\mathbf{B}$. Fédération Internationale de Laiterie. Norme Internationale FIL-IDF 73.

Baird-PARKer (A. C.) (1979). - Methods for identifying Staphylococci and Micrococci. In "Identification methods for Microbiologists ", 2d ed., Academic Press, Londres.

BeERENS (H.) et TAHON-CASTEL (M. M.) (1966). - Milieu à l'acide nalixique pour l'isolement des streptocoques, D. pneumoniae, Listeria, Erysipelotrix. Ann. Inst. Pasteur, III, 90-93.

CARINI (S.), BiAnco (F.) e BARTOLI (I.) (1978). - Il genere aeinetobacter causa di inconvenienti nei formaggi molli. Latte, 3, 257-263.

Duclaux (E.) (1883). - Principe de laiteries. A. Colin et Cie éd., Paris.

Dumont (J. P.), Roger (S.) et AdDA (J.) (1976). - L'arôme du Camembert : autres composés mineurs mis en évidence. Le Lait, 56, 595-599.

Hemme (D.), Boulllanne (C.), Metro (F.) and Desmazeaud (M. J.) (1982). - Microbial catabolism of amino acids during cheese ripening. Sciences des Aliments, 2, 113-123.

HenRiksen (S. D.) (1973). - Moraxella, Acinetobacter, and the Mimea. Bacteriological Review, 17, 522-561.

JACQUet (J.) et COIFFIER (O.) (1973). - Sur les coliformes des fromages à pâte molle. C.R. Société de Biologie de Caen, 167, 39-44.

LAutrop (H.) (1974). - Gram-negative cocci and coccobacilli. Genus III Moraxella. In "Bergey's Manual of Determinative Bacteriology ", 8d ed. The Williams and Wilkins Co, Baltimore (U.S.A.).

Lenoir (J.) (1963). La flore microbienne de Camembert et son évolution au cours de la maturation. Le Lait, 43, 262-270.

Lenoir (J.) et Auberger (B.) (1966). - Contribution à l'étude de la flore microbienne du fromage du type Camembert. 17e Congr. Int. Lait., D2, 595-602.

Maze (P.) (1910). - Technique fromagère. Théorie et pratique. Ann. Inst. Pasteur, 24, 395-428.

Mulder (E. G.) et Antheunisse (J.) (1963). - Morphologie, physiologie et écologie des Arthrobacter. Ann. Microbiol. (Inst. Pasteur), 105, $46-74$.

Mulder (E. G.), Adamse (A. D.), Antheunisse (J.), Deinema (M. H.) Woldendorp (J. W.) and Zevenhuizen (L. P. T. M.) (1966). - The relationship between Brevibacterium linens and bacteria of the genus Arthrobacter. J. appl. Bact., 29, 44-71.

Mourgues (R.), Vassal (L.), Auclair (J.), Mocouot (G.) et Vandeweghe (J.) (1977). Origine et développement des bactéries coliformes dans les fromages à pâte molle. Le Lait, 57, 131-149.

PARK (R. W. A.) and Holding (A. J.) (1966). - Identification of non-fermentive bacilli associated with man. Appl. Microbiol., 16, 1631-1632. 
REYN (A.) (1974). - Gram-negative cocci and coccobacilli. Family I. Neisseriaceae. In "Bergey's Manual of Determinative Bacteriology ", 8d ed. The Williams and Wilkins Co, Baltimore (U.S.A.).

RICHARD (J.) (1980). - Influence de l'agitation du lait cru sur les résultats de dénombrement de sa flore totale à l'aide d'une anse calibrée. Le Lait, 60, 211-255.

Richard (J.) (1982). - Nature de la flore dominante et sous-dominante des laits crus très pollués. Le Lait (soumis pour publication).

RICHARD (J.) et ZADI (H.) (1983). - Classification et tentative d'identification de bactéries corynéformes isolées de Camemberts traditionnels. Annales de Microbiologie (soumis pour publication).

Richard (J.), Krebs (C.), Walter (J.) et Grappin (R.) (1981). - Comparaison de divers modes d'agitation des échantillons de lait cru en vue de la numération de sa flore totale par la méthode Thompson. Le Lait, 61, 250-260.

Rogosa (M.), Cummins (C. S.), Lelliott (R. A.) and Keddie (R. M.) (1974). - Actinomycetes and related organisms. Coryneform group of bacteria. In "Bergey's Manual of Determinative Bacteriology ", 8d ed. The Williams and Wilkins Co, Baltimore (U.S.A.).

Sansonetti (F.) (1930). - Sur les flores bactériennes et fongiques des caillés de lait. Rôle des symbioses dans la maturation du Camembert. Le Lait, 10, 627-640; 782-793; 858-874; 1109-1122.

Tolle (A.), Otte (I.), Suhren (G.), Heeschen (W.) (1980). - Zur Mikroflora von Camembert. Milchwissenschaft, 35, 21-25.

ZADI (H.) (1981). - Etude de la flore bactérienne non lactique des Camemberts fabriqués de façon traditionnelle. Thèse de Docteur-Ingénieur. Université de Paris XI. 